\title{
COVARIANT QUANTUM DYNAMICAL SEMIGROUPS: UNBOUNDED GENERATORS
}

\author{
A.S.Holevo \\ Steklov Mathematical Institute, \\ Vavilova 42, 117966 Moscow, Russia \\ E-mail: holevo@class.mi.ras.ru
}

\begin{abstract}
Summary. A survey of probabilistic approaches to quantum dynamical semigroups with unbounded generators is given. An emphasis is made upon recent advances in the structural theory of covariant Markovian master equations. As an example, a complete characterizations of the Galilean covariant irreversible quantum Markovian evolutions is given in terms of the corresponding quantum master and Langevin equations. Important topics for future investigation are outlined.
\end{abstract}

\section{Introduction}

Quantum dynamical semigroups are a noncommutative analog of (sub-) Markov semigroups in classical probability: while the latter are semigroups of maps in functional spaces, the former are semigroups of maps in operator algebras, having certain properties of positivity and normalization. In quantum statistical mechanics dynamical semigroups arise when one considers weak or singular coupling or low density limits for open quantum system interacting with surrounding, allowing to neglect the memory effects of the interaction [36]. These semigroups satisfy differential equations that are noncommutative generalization of the Fokker-Planck or Chapman-Kolmogorov equations and represent the general solution of the Cauchy problem for such equations.

Let $\mathcal{B}(\mathcal{H})$ be the algebra of all bounded operators in a Hilbert space $\mathcal{H}$. We denote by $I$ the unit operator in $\mathcal{H}$, and by $\operatorname{Id}$ the identity map of $\mathcal{B}(\mathcal{H})$. Since $\mathcal{B}(\mathcal{H})$ is the dual Banach space of the space $\mathcal{T}(\mathcal{H})$ of trace-class operators, it is supplied with the weak topology. On norm-bounded sets this topology coincides with the weak operator topology (see e.g. [6] ). A bounded map $\Phi$ of $\mathcal{B}(\mathcal{H})$ into itself is completely positive (c. p.) if

$$
\sum_{i, j}\left(\psi_{i} \mid \Phi\left[X_{i}^{*} X_{j}\right] \psi_{j}\right) \geq 0
$$

for any finite sets $\left\{\psi_{j}\right\} \in \mathcal{H},\left\{X_{j}\right\} \in \mathcal{B}(\mathcal{H})$. According to Stinespring's theorem adapted to the case of $\mathcal{B}(\mathcal{H})$ (see [30]), a generic weak*-continuous c. p. map has the representation

$$
\Phi[X]=L^{*}\left(X \otimes I_{0}\right) L
$$

where $L$ is a bounded operator from $\mathcal{H}$ to $\mathcal{H} \otimes \mathcal{H}_{0}$ and $I_{0}$ is the unit operator in an auxiliary Hilbert space $\mathcal{H}_{0}$. By using this representation, it is possible to show that such maps satisfying additional normalization condition $\Phi[I]=I$ represent irreversible 
evolutions of the open quantum system interacting via unitary operator with auxiliary system in a fixed initial state (see [30, 15]).

If $I_{0}=\int\left|\psi_{x}><\psi_{x}\right| \mu(d x)$ is a resolution of identity in $\mathcal{H}_{0}$, then (2) implies

$$
\Phi[X]=\int L(x)^{*} X L(x) \mu(x),
$$

where $L(x)=\left(I \otimes \psi_{x}\right)^{*} L$, and $I \otimes \psi_{x}$ is the operator from $\mathcal{H}$ to $\mathcal{H} \otimes \mathcal{H}_{0}$, mapping $\psi$ into $\psi \otimes \psi_{x}$. In particular, if the measure $\mu(d x)$ is discrete, we obtain the familiar representation of a normal c. p. map in $\mathcal{B}(\mathcal{H})$.

By a dynamical semigroup in $\mathcal{B}(\mathcal{H})$ (or quantum dynamical semigroup) we shall call a semigroup $\Phi_{t} ; t \geq 0$, of weak* continuous completely positive maps in $\mathcal{B}(\mathcal{H})$, satisfying $\Phi_{0}=\mathrm{Id}$, and $\Phi_{t}[I] \leq I$. Moreover, for any $X$ the function $t \rightarrow \Phi_{t}[X]$ is required to be weak ${ }^{*}$-continuous. $\Phi_{t}$ is called unital if $\Phi_{t}[I]=I$.

In the case of finite-dimensional $\mathcal{H}$ the weak* continuity is equivalent to the norm continuity; every quantum dynamical semigroup then has the form $\Phi_{t}=\exp t \mathcal{L}$, where $\mathcal{L}$ is the generator of the semigroup. The generator is conditionally completely positive map, which means that inequality of the type (1) holds provided $\sum_{j} X_{j} \psi_{j}=0$, and satisfies the normalization condition $\mathcal{L}[I] \leq 0$ (or $\mathcal{L}[I]=0$ for unital semigroup). The semigroup is the unique solution of the backward and the forward Markovian master equations (M. m. e.)

$$
\frac{d}{d t} \Phi_{t}=\mathcal{L} \circ \Phi_{t} ; \quad \frac{d}{d t} \Phi_{t}=\Phi_{t} \circ \mathcal{L}
$$

satisfying $\Phi_{0}=\mathrm{Id}$.

The conditional complete positivity of $\mathcal{L}$ is equivalent to the standard representation

$$
\mathcal{L}[X]=\Phi[X]-K^{*} X-X K,
$$

where $\Phi$ is a c. p. map of the form (2), and the normalization condition is equivalent to $L^{*} L \leq K^{*}+K$ (with equality for unital semigroups). Similar results hold for norm-continuous semigroups in infinite-dimensional $\mathcal{H}$ with all operators in question being bounded. The representation (4) for this case was established by Lindblad [31, and independently an equivalent representation was obtained by Gorini, Kossakowski and Sudarshan [18 for $\operatorname{dim} \mathcal{H}<\infty$. A physical interpretation for the standard representation can be seen from the Dyson expansion of the solution of the forward M. m. e.

$$
\Phi_{t}=\hat{\Phi}_{t}+\sum_{n=1}^{\infty} \int \ldots \int_{0 \leq t_{1} \leq \ldots \leq t_{n} \leq t} \hat{\Phi}_{t_{1}} \circ \Phi \circ \hat{\Phi}_{t_{2}-t_{1} \ldots} \ldots \circ \hat{\Phi}_{t-t_{n}} d t_{1} \ldots d t_{n}
$$

described as sequence of "spontaneous jumps" of the magnitude $\Phi$ occuring at times $t_{1} \leq \ldots \leq t_{n}$ on the background of the "relaxing evolution" given by the semigroup $\hat{\Phi}_{t}[X]=\mathrm{e}^{-K^{*} t} X \mathrm{e}^{-K t}$.

Let $g \rightarrow V_{g}$ be a unitary representation of a group $G$ in $\mathcal{H}$. The dynamical semigroup $\Phi_{t}$ is called covariant if

$$
\Phi_{t}\left[V_{g}^{*} X V_{g}\right]=V_{g}^{*} \Phi_{t}[X] V_{g}
$$

The property of covariance reflects presence of certain symmetries in the interacting open quantum system and is important for applications. For example, covariance with respect 
to various subgroups of the orthogonal group is characteristic for Bloch type equations and is relevant for optical or magnetic resonance spectroscopy [1] or theory of anisotropic relaxation of spin systems [2]. The combination of covariance and complete positivity imposes strong restrictions on the generator, in some cases defining practically uniquely the form of the corresponding M. m. e.. It can be shown 20] that the generator of a covariant norm continuous semigroup admits the representation (4) in which $\Phi$ is covariant and $K$ commutes with $V_{g}$ (provided $G$ is amenable). This in general is not true for nonnorm continuous case, see Section 4.

Already in the mid-seventies when the structure of norm-continuous dynamical semigroups was well understood, it became clear that the non-norm continuous case, while being interesting from both physical and mathematical points of views, poses difficult problems. The generator of such a semigroup may be unbounded with domain not even necessarily being a ${ }^{*}$ - algebra. Therefore it was difficult to formulate a generalization of conditional complete positivity (or equivalent property) useful enough to obtain a kind of standard representation for $\mathcal{L}$ with all its important consequences. The very formulation of the standard representation needed clarification and it was not obvious whether there are "non-standard" unbounded generators. Among very few papers on the subject, Davies [12] established a standard representation for semigroups on $\mathcal{B}(\mathcal{H})$ having invariant pure state. Bratteli et al. [6] studied semigroups on rather general $\mathrm{C}^{*}$-algebra covariant with respect to a compact Abelian group and satisfying rather strong restriction that $\mathcal{L}$ vanishes on the fixed point subalgebra, and showed a kind of Levy-Khinchin formula for $\mathcal{L}$. There were few papers on quasi-free dynamical semigroups on CCR algebra, generators of which are certainly unbounded and standard, e. g. [14], [37]. Unbounded generators arise when the semigroup is covariant with respect to a non-compact symmetry group (such as translations or Galilei group, [3]). While enormous attention was paid to the study of reversible evolutions generated by Schrödinger operators, much less is known about their irreversible Markovian counterparts.

A substantial progress in this direction was achieved in the past few years by making use of profound analogies from the classical theory of Markov semigroups, as developed by Feller, Dynkin, Ito and McKeane, see e. g. [16], [28], or by direct use of classical probabilistic methods. This development concerns the following topics:

- The minimal dynamical semigroup [11], [9], [10], [21], [33]. Existence and uniqueness of solutions of Markovian master equations [24], [26];

- The structure of (covariant) Markovian master equations [21], [22], [24], [25];

- Noncommutative excessive functions and arrival times [4, 23. Non-standard generators [23], [27];

- Stochastic representations and hyperdissipativity [26]. Relations to continuous measurement processes and nonlinear stochastic Schrödinger equations [17], 26], 229]. Dilations to quantum Langevin equations [25].

In what follows we shall concentrate on the second topic, restricting to brief comments concerning other topics; further details can be found in the references given above. 


\section{The quantum Markovian master equations}

The starting point of our approach, just as in the classical probability theory, is not a semigroup itself, but the differential equation it satisfies. This is also more natural for physical applications. A quantum M. m. e. must be an equation for matrix elements of the semigroup; thus we assume that there is a dense domain $\mathcal{D} \subset \mathcal{H}$, such that the following derivative

$$
\frac{d}{d t}<\psi\left|\Phi_{t}[X] \phi>\right|_{t=0}=\mathcal{L}(\psi ; X ; \phi)
$$

exists for $\phi, \psi \in \mathcal{D}, X \in \mathcal{B}(\mathcal{H})$. The form $\mathcal{L}(\psi ; X ; \phi)$ is called form-generator. It can be characterized by a number of nice properties including conditional complete positivity [21], [24]. These properties turn out to be equivalent to the standard representation

$$
\mathcal{L}(\psi ; X ; \phi)=<L \psi\left|\left(X \otimes I_{0}\right) L \phi>-<K \psi\right| X \phi>-<\psi \mid X K \phi>,
$$

where $L, K$ are (unbounded) operators defined on $\mathcal{D}$ and satisfying the dissipativity condition

$$
\|L \psi\|^{2} \leq 2 \operatorname{Re}<\psi \mid K \psi>, \quad \psi \in \mathcal{D} .
$$

In particular, $K$ is accretive: $\operatorname{Re}\langle\psi| K \psi>\geq 0, \psi \in \mathcal{D}$. The (backward) M. m. e. takes the form

$$
\frac{d}{d t}<\psi \mid \Phi_{t}[X] \phi>=\mathcal{L}\left(\psi ; \Phi_{t}[X] ; \phi\right) ; \quad \phi, \psi \in \mathcal{D} .
$$

The relation between the form-generator and the generator resembles relation between a formal differential operator and its closed extensions determined by certain boundary conditions. To see this let $\Psi_{t}=\left(\Phi_{t}\right)_{*}$ be the strongly continuous preadjoint semigroup in $\mathcal{T}(\mathcal{H})$, such that $\Psi_{t}{ }^{*}=\Phi_{t}$. Denoting its generator $\mathcal{L}_{*}$, one has

$$
\mathcal{L}(\psi ; X ; \phi)=\operatorname{Tr} \mathcal{L}_{*}[|\phi><\psi|] X
$$

for $\phi, \psi \in \mathcal{D}, X \in \mathcal{B}(\mathcal{H})$. The assumption that the derivative (7) exists for all $X$ is equivalent to $\operatorname{dom} \mathcal{L}_{*} \supset \mathrm{D}$, where

$$
\mathrm{D}=\operatorname{lin}\{|\phi><\psi|: \quad \phi, \psi \in \mathcal{D}\}
$$

is a dense domain in $\mathcal{T}(\mathcal{H})$. The M. m. e. (9) takes the form

$$
\frac{d}{d t} \operatorname{Tr} \rho \Phi_{t}[X]=\operatorname{Tr} \mathcal{L}_{*}[\rho] \Phi_{t}[X], \quad \rho \in \mathrm{D}, X \in \mathcal{B}(\mathcal{H}) .
$$

If $\mathrm{D}$ is a core for $\mathcal{L}_{*}$, then this equation determines $\Phi_{t}$ uniquely, otherwise it may have non-unique solution. Under the condition that the closure of $K$ is maximal accretive, one can show that there exists a dynamical semigroup $\Phi_{t}{ }^{\infty}$ giving the minimal solution of the equation (9) in the sense that for any other solution $\Phi_{t}$ the difference $\Phi_{t}-\Phi_{t}{ }^{\infty}$ is completely positive. Of special interest is the case of a unital generator, satisfying $\operatorname{Tr} \mathcal{L}_{*}[\rho] \equiv 0, \rho \in \mathrm{D}$, or

$$
\|L \psi\|^{2}=2 \operatorname{Re}<\psi \mid K \psi>, \quad \psi \in \mathcal{D} .
$$

In general $\Phi_{t}{ }^{\infty}$ may not be unital; however if it is, then $\Phi_{t}{ }^{\infty}$ is the unique solution of (9). 
The method of construction of the minimal dynamical semigroup developed in 11 for resolvents, in [9], [10] for associated integral equation, and in [21], 24] for the backward M. m. e., is the noncommutative extension of the Feller's method [16]. It is based on a standard representation, i. e. on a decomposition of the relevant object into completely positive and relaxing parts. The starting point is the relaxing semigroup $\hat{\Phi}_{t}[X]=\mathrm{e}^{-\bar{K}^{*} t} X \mathrm{e}^{-\bar{K} t}$ providing the unique solution of the equation (9) with

$$
\mathcal{L}(\psi ; X ; \phi)=-<K \psi|X \phi>-<\psi| X K \phi>
$$

which is then perturbed with the completely positive form $<L \psi \mid\left(X \otimes I_{0}\right) L \phi>$ introducing spontaneous jumps on the background of the relaxing evolution $\hat{\Phi}_{t}$. It may be viewed upon as a generalization of the expansion (5) to the case of unbounded but completely positive perturbations. Just as in the classical case, "explosion" may occur if the infinite number of jumps happens during finite interval and the process reaches "boundary" in a finite time (this can never happen for a bounded generator). If $\Phi_{t}^{\infty}$ is not unital, then there is a positive probability of explosion, and additional "boundary conditions" are required to specify the solution, which amounts to certain maximal extension of $\mathcal{L}_{*}$ from $\mathrm{D}$.

Under the additional assumption that operator $L^{*}$ satisfies

$$
\sum_{j}\left\|L^{*}\left(\psi \otimes e_{j}\right)\right\|^{2}<\infty, \quad \psi \in \mathcal{D}^{*}
$$

where $\left\{e_{j}\right\}$ is an orthonormal basis in $\mathcal{H}_{0}$, and $\mathcal{D}^{*} \subset \operatorname{dom} K^{*}$ is a dense domain in $\mathcal{H}_{0}$, one can write also the forward Markovian master equation for the preadjoint semigroup $\Psi_{t}$ :

$$
\frac{d}{d t}<\phi \mid \Psi_{t}[\rho] \psi>=\mathcal{L}_{*}\left(\phi ; \Psi_{t}[\rho] ; \psi\right) ; \quad \phi, \psi \in \mathcal{D}^{*}
$$

where

$$
\begin{gathered}
\mathcal{L}_{*}(\phi ; \rho ; \psi)=\operatorname{Tr} \rho \mathcal{L}[|\psi><\phi|] \\
=\sum_{j}<L^{*}\left(\phi \otimes e_{j}\right)\left|\rho L^{*}\left(\psi \otimes e_{j}\right)>-<K^{*} \phi\right| \rho \psi>-<\phi \mid \rho K^{*} \psi>,
\end{gathered}
$$

and $\mathcal{L}$ is the generator of $\Phi_{t}$ defined on $\mathrm{D}^{*}=\left\{|\psi><\phi|: \phi, \psi \in \mathcal{D}^{*}\right\}$. Assuming $K^{*}$ to be maximal accretive one can prove that $\Psi_{t}^{\infty}=\left(\Phi_{t}{ }^{\infty}\right)_{*}$ is the minimal solution of the forward equation [25], [26]. However in general the forward and the backward equations are no longer equivalent. Thus the situation is similar to that for the Kolmogorov-Feller differential equations in the theory of Markov processes [16].

Going back to the problem of standard representation, we can make the following remarks. The fact that a form-generator has the standard representation (8) implies the possibility of decomposing the generator $\mathcal{L}_{*}$ into completely positive and relaxing parts only on the subspace D which need not be a core for $\mathcal{L}_{*}$. If explosion occurs, these two parts need not be separately extendable onto a core for $\mathcal{L}_{*}$. On the other hand, generators of different dynamical semigroups restricted to $\mathrm{D}$ can give rise to one and the same standard expression (8). One may formalize the notion of standard representation by saying that a dynamical semigroup is standard if it can be constructed as the minimal semigroup for some M. m. e., that is by a completely positive perturbation of a relaxing semigroup. In [23 a possible noncommutative extension of "boundary conditions" for conservative 
form-generator was proposed as very singular completely positive perturbations vanishing on the dense domain D. By using such a perturbation the author gave a construction of non-standard dynamical semigroup on $\mathcal{B}(\mathcal{H})$ [23], 27].

\section{An example}

Let $\xi_{t}, t \geq 0$ be stochastic process with stationary independent increments [16]. Roughly speaking, the (generalized) time derivative of $\xi_{t}$ is a continuous analog of a sequence of independent identically distributed random variables, that is a classical "noise" process. One of the beautiful results of probability theory is the Levy-Khinchin formula describing the possible form of the characteristic function of such process:

$$
\operatorname{Mexp} i \lambda \xi_{t}=\exp t\left[i \beta \lambda-\frac{\alpha}{2} \lambda^{2}+\int_{0<|y|}\left(\mathrm{e}^{i y \lambda}-1-i y \lambda 1_{h}(y)\right) \mu(d y)\right]
$$

where $\beta$ is real number, $\alpha \geq 0, h$ is arbitrary but fixed positive number, $1_{h}(y)$ is the indicator of the set $|y| \leq h$, and $\mu(d y)$ is a positive measure on the set $\mathbf{R} \backslash\{0\}$, satisfying the condition

$$
\int_{0<|y|}\left[y^{2} 1_{h}(y)+\left(1-1_{h}(y)\right)\right] \mu(d y)<\infty .
$$

In (13) the term $i \beta \lambda-\frac{\alpha}{2} \lambda^{2}$ corresponds to the Gaussian component of the process $\xi_{t}$, which is a continuous process. If in the integral term we take $\mu(d y)=\mu \delta\left(y-y_{0}\right) d y$ with $\mu>0$, then for $\left|y_{0}\right|>h$ we obtain logarithm of the characteristic function of the Poisson process with the jumps of the magnitude $y_{0}$. Therefore for arbitrary measure $\mu(d y)$ the integral $\int_{h<|y|}\left(\mathrm{e}^{i y \lambda}-1\right) \mu(d y)$ describes the mixture of independent Poisson processes with various magnitudes $y,|y|>h$. It corresponds to the discontinuous (pure jump) component of the process $\xi_{t}$ (with magnitudes of jumps $|y|>h$ ). The value of $h$ is arbitrary but fixed, so the name "big jumps" is only conventional. The term related to "small jumps" (of magnitudes $|y| \leq h$ ) corresponds to the situation when infinitely many small jumps can accumulate during finite time, and one must include portions of linear drift between jumps in order that the total increment will remain finite. The process $\xi_{t}$ itself can be decomposed into three components - continuous Gaussian, Poisson "big jumps" and "small jumps", according to Ito's formula (see e. g. [32]):

$$
d \xi_{t}=\beta d t+\sqrt{\alpha} d W_{t}+\int_{h<|y|} y \Pi(d y d t)+\int_{0<|y| \leq h} \tilde{\Pi}(d y d t)
$$

where $W_{t}$ is the standard Wiener process, $\Pi(d y d t)$ is the Poisson random measure on $\mathbf{R}^{2}$ with the compensator $\mu(d y) d t$, so that

$$
\mathrm{M} d W_{t}=0, \quad \mathrm{M}(d y d t)=\mu(d y) d t
$$

and $\tilde{\Pi}(d y d t)=\Pi(d y d t)-\mu(d y) d t$ is the compensated random measure. Note that $\Pi\left(\left[y_{1}, y_{2}\right],\left[t_{1}, t_{2}\right]\right)$ is just the number of jumps of the process $\xi_{t}$ on the time interval $\left[t_{1}, t_{2}\right]$, which have magnitudes $y \in\left[y_{1}, y_{2}\right]$.

Now consider the Hilbert space $\mathcal{H}=L^{2}(\mathbf{R})$, and let $Q=x, P=i^{-1} \frac{d}{d x}$ be, respectively, the self-adjoint position and momentum operators for one-dimensional quantum system, 
so that $V_{y}=\exp (i y Q), y \in \mathbf{R}$, and $U_{x}=\exp (-i x P), x \in \mathbf{R}$, are the unitary groups in $\mathcal{H}$ satisfying the Weyl canonical commutation relation $(\mathrm{CCR})$ :

$$
U_{x} V_{y}=\exp (-i x y) V_{y} U_{x}
$$

Defining

$$
\Phi_{t}[X]=\mathrm{M}_{\xi_{t}}^{*} X U_{\xi_{t}}, \quad t \geq 0,
$$

one easily sees that $\Phi_{t}$ is a unital dynamical semigroup in $\mathcal{H}$. Indeed, operators $\Phi_{t}$ are manifestly completely positive; the semigroup property follows from the fact that $\xi_{t}$ has stationary independent increments; the weak* continuity properties follow from the continuity properties of $U_{x}$ and of the expectation. The semigroup (18) represents the dynamics of quantum system in $\mathcal{H}$ interacting with the classical noise via unitary operators $\exp \left(-i \xi_{t} P\right)$, averaged with respect to the distribution of the noise. To find the generator of this semigroup, one can use the Ito formula for $\exp \left(i \xi_{t} P\right)$ (cf. [19]):

$$
\begin{aligned}
& d \exp \left(i \xi_{t} P\right)=\exp \left(i \xi_{t} P\right)\left\{\left[i \beta P-\frac{\alpha}{2} P^{2}+\int_{0<|y| \leq h}(\exp (i y P)-1-i y P) \mu(d y)\right] d t\right. \\
& \left.+i \sqrt{\alpha} P d W_{t}+\int_{h<|y|}[\exp (i y P)-1] \Pi(d y d t)+\int_{0<|y| \leq h}[\exp (i y P)-1] \tilde{\Pi}(d y d t)\right\},
\end{aligned}
$$

and the Ito product rule

$$
d W_{t}^{2}=d t, \quad \Pi(d y d t)^{2}=\Pi(d y d t),
$$

with all other products of stochastic differentials (including $d t$ ) equal to zero. Taking into account (16), one can obtain both backward and forward M. m. e. (9), (12) with $\mathcal{D}=\mathcal{D}^{*}=C_{0}^{2}(\mathbf{R})$, the subspace of twice continuously differential functions with compact support, where the form-generators correspond to the expression

$$
\mathcal{L}[X]=i \beta[P, X]-\frac{\alpha}{2}[P,[P, X]]+\int_{0<|y|}\left(U_{y}^{*} X U_{y}-X-i y[P, X] 1_{h}(y)\right) \mu(d y),
$$

defined for $X \in \mathrm{D}$. Here the first term is the Hamiltonian "drift", the second term corresponds to the interaction with the Gaussian "white" noise and is typical for diffusion approximations, while the last term reflects the influence of the Poisson "shot" noises arising in low density limits. The generator (20) is bounded if and only if $\alpha=0, \beta=0$ and $\mu(d y)$ is a finite measure on $\mathbf{R} \backslash 0$.

The Gaussian noise gives rise to the diffusive generator

$$
\mathcal{L}[X]=-\frac{\alpha}{2}[P,[P, X]]=\frac{\alpha}{2}\left(2 P X P-P^{2} X-X P^{2}\right),
$$

with the obvious standard representation on D. A standard representation for the last term in (20) can be obtained by taking

$$
L=\int_{0<|y|}(I \otimes \mid y>)\left(U_{y}-I\right) \mu(d y), \quad K=\int_{0<|y|}\left(I-U_{y}-i y P 1_{h}(y)\right) \mu(d y),
$$

where $\{\mid y>\}$ is the canonical family of "ket" vectors in $\mathcal{H}_{0}=L^{2}(\mathbf{R}, \mu)$. 
From the CCR it follows that the semigroup is covariant with respect to the representation $y \rightarrow V_{y}$ describing translations in the momentum space. As shown in [21], for covariant M. m. e. the non-explosion in $\mathcal{B}(\mathcal{H})$ is equivalent to the non-explosion in the fixed-point subalgebra $\mathcal{A}_{V}=\left\{X: V_{g}^{*} X V_{g}=X, g \in G\right\}$ of the representation $g \rightarrow V_{g}$. If this subalgebra is Abelian then the problem is reduced to the well-studied problem of non-explosion for a classical Markov process. In our example the fixed point algebra is the maximal Abelian subalgebra $\mathcal{A}_{Q}$ of operators of the form $X=f(Q)$; by the CCR

$$
\Phi_{t}[f(Q)]=\mathrm{M} f\left(Q+\xi_{t}\right),
$$

and

$$
\mathcal{L} f(x)=\beta \frac{d f(x)}{d x}+\frac{\alpha}{2} \frac{d^{2} f(x)}{d x^{2}}+\int_{0<|y|}\left[f(x+y)-f(x)-y f^{\prime}(x) 1_{h}(y)\right] \mu(d y)
$$

is the generator of the semigroup corresponding to the process $\xi_{t}$ with stationary independent increments, for which explosion can never occur [16]. This is also strictly related to the additional property of covariance with respect to the space translations $x \rightarrow U_{x}$, shared by the semigroup (18). However the situation is different for more general momentum translation covariant M. m. e..

To see this, following [24], consider the Hilbert space $\mathcal{H}=L^{2}(l, \infty)$, the domain $\mathcal{D}=$ $C_{0}^{2}(l, \infty)$ consisting of continuously twice differentiable functions with compact support, vanishing at $l$, and the form-generator

$$
\mathcal{L}(\phi, X, \psi)=<(P+L(Q)) \phi \mid X(P+L(Q) \psi>-<K \phi|X \psi>-<\phi| X K \psi>,
$$

defined for $\phi, \psi \in \mathcal{D}$, where $K=\frac{P^{2}}{2}+P L(Q)+\frac{|L(Q)|^{2}}{2}$, and $L(Q)$ is a continuously differentiable complex function. This form-generator is covariant with respect to the representation $y \rightarrow V_{y}=\exp (i y Q)$, and hence the corresponding minimal dynamical semigroup is also covariant [21]. The restriction to the fixed point algebra $\mathcal{A}_{Q}$ corresponds to the classical diffusion on $(l, \infty)$ with the generator

$$
\mathcal{L} f(x)=2 \operatorname{Im} L(x) \frac{d f(x)}{d x}+\frac{1}{2} \frac{d^{2} f(x)}{d x^{2}} .
$$

Non-explosion means that both $l$ and $\infty$ are non-absorbing boundaries for this diffusion. The necessary and sufficient condition for this is Feller's test [28], saying that the function

$$
\int_{x_{0}}^{x}\left[\exp \int_{x}^{y} 4 \operatorname{Im} L(z) d z\right] d y
$$

where $x_{0} \in(l, \infty)$, must be non-integrable in the neighbourhoods of both $l$ and $\infty$. In particular, if $L(x) \equiv 0$ (pure diffusion with no drift), then the probability of absorption at $l$ is positive, hence the minimal semigroup is non-unital and the solution of the M. m. e. is not unique. This minimal semigroup is the extension onto $\mathcal{B}(\mathcal{H})$ of the Markov semigroup corresponding to the Brownian motion on $(l, \infty)$ killed at the boundary $l$. Other solutions of the backward M. m. e. are obtained by taking perturbations corresponding to various boundary conditions at $l$. An example of non-standard dynamical semigroup on $\mathcal{B}(\mathcal{H})$ is constructed as a singular perturbation of this minimal semigroup corresponding to rebounding from $l$ to a fixed quantum state $\rho_{0}$ [23], [27]. 


\section{Covariant evolutions and the group cohomology}

Consider a backward M. m. e. given by a form-generator $\mathcal{L}(\phi, X, \psi)$. The standard representation (8) of the form-generator is not unique even if it is subjected to further condition of minimality [24]. If $D$ is a unitary operator in $\mathcal{H}_{0}, a \in \mathcal{H}_{0}$, and $b$ is a real number, then the operators

$$
L^{\prime}=(I \otimes D) L+I \otimes a, \quad K^{\prime}=K+(I \otimes a)^{*}(I \otimes D) L+\left[\frac{1}{2}\|a\|^{2}-i b\right] I,
$$

where $(I \otimes a)$ is the operator from $\mathcal{H}$ to $\mathcal{H} \otimes \mathcal{H}_{0}$ acting as $(I \otimes a) \psi=\psi \otimes a$, give another standard representation for $\mathcal{L}(\phi, X, \psi)$ satisfying the minimality condition. The transformations $(D, a, b):(L, K) \rightarrow\left(L^{\prime}, K^{\prime}\right)$ form a kind of a "gauge group" (cf. also [34) under the multiplication law

$$
\left(D^{\prime}, a^{\prime}, b^{\prime}\right)(D, a, b)=\left(D^{\prime} D, D^{\prime} a+a^{\prime}, b+b^{\prime}-\operatorname{Im}<a^{\prime} \mid D^{\prime} a>\right) .
$$

We denote this group by $G(\mathcal{L})$. It is endowed with the natural topology as a subset of the product $\mathcal{U}\left(\mathcal{H}_{0}\right) \times \mathcal{H}_{0} \times \mathbf{R}$, where $\mathcal{U}\left(\mathcal{H}_{0}\right)$ is the group of unitary operators in $\mathcal{H}_{0}$ with the weak operator topology.

Let now the form-generator be covariant under a (projective) unitary representation $g \rightarrow V_{g}$ of a symmetry group $G$, namely, the domain $\mathcal{D}$ be invariant under $V_{g}$ and

$$
\mathcal{L}\left(\phi, V_{g}^{*} X V_{g}, \psi\right)=\mathcal{L}\left(V_{g} \phi, X, V_{g} \psi\right), \quad \phi, \psi \in \mathcal{D} .
$$

Theorem 1. There is a representation $g \rightarrow\left(D_{g}, a_{g}, b_{g}\right)$ of $G$ in $G(\mathcal{L})$ such that

$$
\left(V_{g}^{*} L V_{g}, V_{g}^{*} K V_{g}\right)=\left(D_{g}, a_{g}, b_{g}\right)(L, K) .
$$

If $V_{g}$ is a continuous representation of a topological group $G$ then the representation $\left(D_{g}, a_{g}, b_{g}\right)$ is continuous if and only if the scalar function $g \rightarrow \mathcal{L}\left(\phi, X V_{g}^{*}, V_{g} \psi\right)$ is continuous for all $\phi, \psi \in \mathcal{D}, X \in \mathcal{B}(\mathcal{H})$.

The proof of this theorem may be found in [24], and here we discuss briefly the way it can be applied to find the form of a covariant generator for concrete symmetry groups $G$. From this theorem taking into account (24) it follows that $D_{g}$ is a unitary representation of $G$ and $a_{g}$ is a first order cocycle for this representation in $\mathcal{H}_{0}: a_{g^{\prime} g}=D_{g^{\prime}} a_{g}+a_{g^{\prime}}$. Moreover, the real function $b_{g}$ satisfies the coboundary equation $b\left(g^{\prime}\right)+b(g)-b\left(g^{\prime} g\right)=$ $\operatorname{Im}<a_{g^{\prime}} \mid D_{g^{\prime}} a_{g}>$. Thus the structure of the covariant form-generator is determined by the low order cohomology of the group $G$, which was studied in detail for many interesting groups (see, e. g. [35]). In particular, it is well known that the low order cohomology is trivial for compact groups, that is every cocycle is a coboundary for such groups, $a_{g}=\left(D_{g}-I\right) a$ for some $a \in \mathcal{H}_{0}$. It follows that in this case, similarly to the case of bounded generators, the covariant form-generator has the standard representation (8) where the c. p. component and the relaxing terms are separately covariant. That this is not the case for non-compact groups, can be easily seen from the example of the diffusive generator (21). 


\section{Galilean covariant Markovian evolutions}

Let $(\xi, \tau) \in \mathbf{R}^{2}$ be a point in the 2-dimensional non-relativistic space-time, and let $(x, v, t):(\xi, \tau) \rightarrow\left(\xi^{\prime}, \tau^{\prime}\right)$ be the Galilei transformation

$$
\xi^{\prime}=\xi+x+v \tau, \quad \tau^{\prime}=\tau+t,
$$

where $x \in \mathbf{R}$ is the space shift, $v \in \mathbf{R}$ the Galilean boost. For simplicity we consider zero-spin unit mass elementary system characterized by the Weyl operators

$$
W_{x, v}=\exp i(v Q-x P)=V_{v} U_{x} \exp \left(\frac{i}{2} v x\right),
$$

constituting irreducible representation of the CCR.

A dynamical semigroup $\Phi_{t}$ is Galilean covariant [3], [25], if

$$
\Phi_{t}\left[W_{x, v}^{*} X W_{x, v}\right]=W_{x-v t, v}^{*} \Phi_{t}[X] W_{x-v t, v} .
$$

Let $\mathcal{D} \subset \mathcal{H}$ be the dense domain

$$
\mathcal{D}=\bigcap_{x, v \in \mathbf{R}} \operatorname{dom}(v Q-x P)^{2},
$$

and let $\mathrm{D} \subset \mathcal{B}(\mathcal{H})$ be the domain defined by the relation (10). We remark that $\mathcal{D}$ is invariant under $W_{x, v}$, and that $\mathrm{D}$ is norm-dense in $\mathcal{T}(\mathcal{H})$ and weakly ${ }^{*}$ - dense in $\mathcal{B}(\mathcal{H})$. We make the following assumption

(A) The domain $\mathrm{D}$ is contained both in $\operatorname{dom} \mathcal{L}_{*}$ and $\operatorname{dom} \mathcal{L}$.

Theorem 2. A unital Galilean covariant dynamical semigroup satisfying the condition (A) has the generator $\mathcal{L}$ given by the following expression on $\mathrm{D}$

$$
\begin{gathered}
\mathcal{L}[X]=i\left[\frac{P^{2}}{2}, X\right]+i\left[\beta_{P} P+\beta_{Q} Q, X\right] \\
-\frac{1}{2}\left\{\alpha_{P P}[P,[P, X]]+\alpha_{P Q}[P,[Q, X]]+\alpha_{Q Q}[Q,[Q, X]]\right\} \\
+\iint_{x^{2}+v^{2}>0}\left\{W_{x, v}^{*} X W_{x, v}-X-i[x P-v Q, X] 1_{h}(x, v)\right\} \nu(d x d v),
\end{gathered}
$$

where $\beta_{P}, \beta_{Q} \in \mathbf{R}$, the real matrix

$$
\left[\begin{array}{ll}
\alpha_{P P} & \alpha_{P Q} \\
\alpha_{P Q} & \alpha_{Q Q}
\end{array}\right]
$$

is positive definite, $1_{h}(x, v)$ is the indicator of the set $x^{2}+v^{2} \leq h$ and $\nu(d x d v)$ is a positive measure on $\mathbf{R}^{2} \backslash\{0\}$ satisfying the Levy condition

$$
\iint_{x^{2}+v^{2}>0}\left\{\left(x^{2}+v^{2}\right) 1_{h}(x, v)+\left[1-1_{h}(x, v)\right]\right\} \nu(d x d v)<\infty .
$$

Moreover, the domain D is a core for both $\mathcal{L}_{*}$ and $\mathcal{L}$ and the corresponding M. m. e. (9), (12) have $\Phi_{t}\left(\right.$ resp. $\left.\Psi_{t}\right)$ as the unique solution. 
The last statement of the Theorem applies to particular Galilean covariant M. m. e. arising in various physical applications, such as quantum optics [7], precision experiments [5], nonlinear quantum mechanics [13] etc. The uniqueness of the solution of the M. m. e. is related to the fact that the fixed point algebra of the representation $(x, v) \rightarrow W_{x, v}$ is trivial, that is consists of multiples of the identity operator (cf. 21]).

A derivation of (26) can be based on Theorem 1. By subtracting from $\mathcal{L}$ the Hamiltonian term corresponding to the free motion we obtain a generator $\mathcal{L}_{0}$ satisfying the condition of Weyl covariance

$$
\mathcal{L}_{0}\left[W_{x, v}^{*} X W_{x, v}\right]=W_{x, v}^{*} \mathcal{L}_{0}[X] W_{x, v} .
$$

Let $L, K$ be the components of the standard representation of the corresponding formgenerator. According to Theorem 1 there is a unitary representation $(x, v) \rightarrow D_{x, v}$ of the Abelian group $\mathbf{R}^{2}$ and the cocycle $a_{x, v}$ in the Hilbert space $\mathcal{H}_{0}$ such that $L, K$ satisfy the covariance equations

$$
\begin{gathered}
W_{x, v}^{*} L W_{x, v}=\left(I \otimes D_{x, v}\right) L-I \otimes a_{-x,-v} \\
W_{x, v}^{*} K W_{x, v}=K-\left(I \otimes a_{-x,-v}\right)^{*} L+\left[\frac{1}{2}\left\|a_{x, v}\right\|^{2}-i b_{x, v}\right] I .
\end{gathered}
$$

These equations can be solved by diagonalizing the representation $D_{x, v}$ and by using the structure of cocycles for representations of Abelian locally compact groups [22]. The "Gaussian" part of the generator $\mathcal{L}_{0}$ arises from the identity subrepresentation of $D_{x, v}$ while the orthogonal complement gives the "jump" part. We conjecture that the assumption (A) can be deduced from the Galilean covariance itself, as we were able to deduce it from the Weyl covariance (see [25], where an alternative proof of Theorem 2 is given).

The generator (20) considered in Section 3 is a particular case of (26), provided we exclude the free Hamiltonian term. That generator arose from the semigroup (18) describing interaction of quantum system with the classical noise. It turns out to be possible to give a similar explicit description of the Galilean covariant quantum open systems, as systems interacting with specific classical noises. Let $\xi_{t}, \eta_{t}$ be a classical stochastic process with stationary independent increments in $\mathbf{R}^{2}$, defined by the characteristic function of the Levy-Khinchin form

$$
\begin{gathered}
\operatorname{Mexp} i\left(\mu \xi_{t}-\lambda \eta_{t}\right)=\operatorname{expt}\left\{i\left(\mu \beta_{P}-\lambda \beta_{Q}\right)-\frac{1}{2}\left(\alpha_{P P} \mu^{2}+2 \alpha_{P Q} \mu \lambda+\alpha_{Q Q} \lambda^{2}\right)\right. \\
\left.+\iint_{x^{2}+v^{2}>0}\left[\mathrm{e}^{i(\mu x-\lambda v)}-1-i(\mu x-\lambda v) 1_{h}(x, v)\right] \nu(d x d v)\right\},
\end{gathered}
$$

where $\beta_{P}, \beta_{Q} ; \alpha_{P P}, \alpha_{P Q}, \alpha_{Q Q}$ and $\nu(d x d v)$ are taken from (26). Consider the stochastic differential equations

$$
d Q_{t}=\frac{P_{t}}{m} d t+d \xi_{t}, \quad d P_{t}=d \eta_{t}
$$

with the initial conditions $Q_{0}=Q, P_{0}=P$. These will be the Heisenberg equations for our open quantum system. They correspond to the infinitesimal canonical transformation with the Hamiltonian

$$
d H_{t}=\frac{P^{2}}{2} d t+P d \xi_{t}-Q d \eta_{t}
$$


Defining the chronologically ordered exponential

$$
U_{t}(\xi, \eta)=\mathcal{T} \exp \left(-i \int_{0}^{t} d H_{s}\right)
$$

as the solution of the corresponding stochastic differential equation, we can prove (see 25) that

$$
\Phi_{t}[X]=\mathrm{MU}_{t}(\xi, \eta)^{*} X U_{t}(\xi, \eta)
$$

This relation is a generalization of the representation (18) and the proof proceeds along similar lines by using the stochastic differential equation for $U_{t}(\xi, \eta)$ and the distribution of $\xi_{t}, \eta_{t}$ defined by (27). Equations (28) are the Langevin equation giving the dilation of the dynamical semigroup $\Phi_{t}$ with the classical stationary independent increment processes as the driving noises.

\section{Discussion}

The results described in the previous Section are due to the very restrictive nature of the full Galilean covariance. We obtain much broader and physically interesting class of quantum Markovian evolutions by omitting space translations and restricting only to Galilean boosts, that is to the fundamental symmetry of a non-relativistic particle in a potential field. The class of resulting evolutions is described in detail in 25] for the case where the position space is the whole $\mathbf{R}^{3}$. Discussion at the end of Section 3 suggests that contrary to the case of full Galilean covariance, there is no automatic nonexplosion, and boundary conditions should play an important role, especially for systems with restricted position domains. This case deserves much more detailed study. Other interesting problems are related to introducing spin degrees of freedom along with spatial ones and to gauge covariance.

Another important distinction of the boost covariant evolutions is that they describe open systems interacting with quantum rather than classical noises. This means that the corresponding M. m. e. at least formally can be dilated to the Langevin equations (see [25]) which are quantum stochastic differential equations driven by quantum Brownian motion or Poisson-type processes in the sense of [34], but in general, with unbounded operator coefficients. For example, the Langevin equation dilating the diffusive M. m. e. defined by the form-generator (22) supplemented with the Hamiltonian term has the form

$$
\begin{gathered}
d f\left(Q_{t}\right)=i\left[\frac{P_{t}^{2}}{2}, f\left(Q_{t}\right)\right] d t+f^{\prime}\left(Q_{t}\right) i\left[\left(d A_{t}+L\left(Q_{t}\right) d t\right)^{\dagger}-\text { h. c. }\right]+\frac{1}{2} f^{\prime \prime}\left(Q_{t}\right) d t \\
d P_{t}=U^{\prime}\left(Q_{t}\right) d t+i\left[\bar{L}^{\prime}\left(Q_{t}\right)\left(d A_{t}+\frac{1}{2} L\left(Q_{t}\right) d t\right)-\text { h.c. }\right],
\end{gathered}
$$

where $U$ is the potential, $A_{t}^{\dagger}, A_{t}$ are creation-annihilation processes representing quantum Brownian motion and h. c. denotes hermitean conjugated terms.

Remarkably, at least for the minimal solution of M. m. e. there always exists a representation via solutions of certain classical dissipative stochastic equation in the Hilbert space of the system. It provides a powerful probabilistic tool for study of the problem 
of non-explosion for quantum dynamical semigroups [26], and of the nonlinear stochastic Schrödinger equation arising in the theory of continuous quantum measurement processes [17], [26], [29].

Acknowledgements. The author acknowledges support from Arnold Sommerfeld Institute for Mathematical Physics, Technical University Clausthal, during the XXI International Colloquium on Group Theoretical Methods in Physics. The work was partially supported by RFBR grant no. 96-01-01709.

\section{References}

[1] R. Alicki, K. Lendi, Quantum Dynamical Semigroups and Applications, Lect. Notes. Phys. 286, Springer-Verlag, Berlin-Heidelberg-NY 1987.

[2] A. Yu. Artem'ev, Teor. Mat. Fiz. 87, 34-39 (1991); Application of Methods of Completely Positive Semigroups in Study of Dynamics of Open Spin Systems, Ph. D. Thesis, Moscow 1991.

[3] A. Barchielli, L. Lanz, Il Nuovo Cimento 44B, 241-264 (1978).

[4] B. V. Bhat, K. R. Parthasarathy, Ann. Inst. H. Poincare, ser. B 31, 601-652 (1995).

[5] V. B. Braginsky, F. Ya. Khalili, Quantum Measurements, Cambridge University Press 1992.

[6] O. Bratteli, D. W. Robinson, Operator Algebras and Quantum Statistical Mechanics I, Springer-Verlag, Berlin 1981.

[7] H. J. Carmichael, An Open System Approach to Quantum Optics, Lect. Notes Phys. m18, Springer-Verlag, Berlin-Heidelberg-NY 1993.

[8] O. Bratteli, P. E. T. Jørgensen, A. Kishimoto, D. W. Robinson, Ann. Inst. Fourier 34, 155-187 (1984).

[9] A. M. Chebotarev, J. of Soviet Math. 56, 2697-2719 (1991).

[10] A. M. Chebotarev, F. Fagnola, J. Funct. Anal. 118, 131-153 (1993).

[11] E. B. Davies, Rep. Math. Phys. 11, 169-188 (1977).

[12] E. B. Davies, J. Funct. Anal. 34, 421-432 (1979).

[13] H.-D. Doebner, J. D. Hennig, P. Nattermann, Lecture at the XX International Colloquium on Group Theoretical Physics, Osaka 1994.

[14] G. G. Emch, S. Albeverio, J.-P. Eckmann, Rept. Math. Phys. 13, 73-85 (1978).

[15] D. E. Evans, J. T. Lewis, Dilations of Irreversible Evolutions in Algebraic Quantum Theory, Commun. Dublin Inst. Adv. Studies, Ser. A, 24 Dublin 1977. 
[16] W. Feller, An Introduction to Probability Theory and Its Applications II, J. Wiley, NY (1966).

[17] D. Gatarek, N. Gisin, J. Math. Phys. 32, 2152-2157 (1991).

[18] V. Gorini, A. Kossakowski, E. Sudarshan, Journ. Math. Phys. 17, 821-825 (1976).

[19] A. S. Holevo, Lect. Notes. Math. 1442, 211-215 (1990).

[20] A. S. Holevo, Rept. Math. Phys. 32, 211-216 (1993).

[21] A. S. Holevo, Rept. Math. Phys. 33, 95-110 (1993).

[22] A. S. Holevo, Izvestiya: Mathematics 59:2, 427-443 (1995).

[23] A. S. Holevo, Izvestiya: Mathematics 59:6, 1311-1325 (1995).

[24] A. S. Holevo, J. Funct. Anal. 131, 255-278 (1995).

[25] A. S. Holevo, J. Math. Phys. 37(4), 1812-1832 (1996).

[26] A. S. Holevo, Probab. Theory Rel. Fields 104, 483-500 (1996).

[27] A. S. Holevo, Uspekhi Mat. Nauk. 51(6), 225-226 (1996).

[28] K. Ito, H. P. McKeane, Diffusion Processes and Their Sample Paths, Springer-Verlag, Berlin-Heidelberg-NY 1965.

[29] V. N. Kolokoltsov, J. Math. Phys. 36, 2741-2760 (1995).

[30] K. Kraus, States, Effects and Operations, Lect. Notes Phys. 190, Springer-Verlag, Berlin-Heidelberg-NY 1983.

[31] G. Lindblad, Commun. Math. Phys. 48, 119-130 (1976).

[32] R. Sh. Liptser, A. N. Shiryaev, Theory of Martingales, Kluwer, Dodrecht 1986.

[33] A. Mohari, K. B. Sinha, Proc. Indian Acad. Sci., 102, 159-173 (1992).

[34] K. R. Parthasarathy, An Introduction to Quantum Stochastic Calculus, Birkhäuser Verlag, Basel-Boston-Berlin 1992.

[35] K. R. Parthasarathy, K. Schmidt, Positive Definite Kernels, Continuous Tensor Products and the Central Limit Theorem of Probability Theory, Lect. Notes Math. 272, Springer-Verlag, Berlin-Heidelberg 1972.

[36] H. Spohn, Rev. Mod. Phys. 53, 569-615 (1980).

[37] P. Vanheuverzwijn, Ann. Inst. H. Poincare, ser. A 19, 123-138 (1978). 